\title{
Usages littéraires de l'apostrophe : fonctions textuelles et pragmatiques et spécificités génériques
}

\author{
Michèle Monte \\ BCL ${ }^{1} /$ Université du Sud Toulon Var \\ monte@univ-tln.fr
}

L'apostrophe à l'allocutaire dans un énoncé oral ou un discours écrit destiné à être prononcé en public est régie par des nécessités pragmatiques complexes. Dans un travail pionnier, Perret (1970) distinguait pour l'apostrophe un rôle d'identification du référent, un rôle prédicatif, lié au sens lexical, et un rôle sociolinguistique, précisant les relations qui unissent locuteur et allocutaire. Détrie (2006) étudie pour sa part le triple rôle interactionnel, textuel et argumentatif de l'apostrophe qui, tour à tour ou simultanément, régule l'activité conversationnelle en ratifiant tel ou tel comme allocutaire, marque les débuts et fins d'échange, évalue l'allocutaire en l'interpellant au moyen de lexèmes subjectifs. Tout en réservant des développements spécifiques à l'apostrophe en discours romanesque ou dans la poésie lyrique, Détrie ne distingue pas radicalement les emplois littéraires des autres emplois. Est-ce à dire que l'apostrophe fonctionne de façon globalement similaire dans des contextes littéraires mimant la communication orale et dans des contextes de communication effective ? Cela méritait de plus amples investigations et c'est pourquoi j'ai voulu comparer des écrits littéraires et des discours oralisés en me proposant de préciser l'influence des contraintes génériques sur le fonctionnement de l'apostrophe. J'ai pour ce faire constitué un corpus (précisé à la fin de cet article) comprenant des dialogues de roman, des textes de théâtre, et des textes poétiques, en utilisant comme témoins d'un usage non littéraire de l'apostrophe des oraisons funèbres et des discours politiques et judiciaires. Je présenterai les résultats obtenus en distinguant d'une part les dialogues de roman et de théâtre, pour lesquels les usages de l'apostrophe ne semblent pas très différents de ce que l'on peut observer dans les discours non littéraires, d'autre part les textes poétiques monologués, qui obéissent à des contraintes spécifiques.

\section{L'apostrophe dans les dialogues de roman et au théâtre}

Précisons tout d'abord que je n'analyserai pas dans ce travail les modifications du contenu sémantique des apostrophes au fil de l'interaction. Plusieurs raisons à cela : tout d'abord, dans le corpus que j'ai constitué, les dénominations restent extrêmement stables. Les personnages s'interpellent le plus souvent par leur prénom, éventuellement précédé de «monsieur » ou "madame» selon des variations qu'il est aisé de justifier : la marque de politesse correspond généralement à l'adoucissement d'un acte menaçant pour une des faces de l'allocutaire. On trouve aussi «Monsieur» ou "Madame » seuls ou suivis d'un titre, ainsi que des termes désignant des métiers ou une position hiérarchique (" pédagogue » et " maitre » dans Les Mouches, par exemple) qui, eux aussi, soit restent stables, soit sont sujets à des variations aisément explicables : «monsieur le Juge » dans Le Balcon alterne avec «monsieur » et correspond à une déférence plus marquée, sollicitée par le personnage lui-même. L'apparition de termes affectifs tels que « mes amis » ou «mon chéri » ou d'insultes telles que «gredin » ou «coquine» s'explique facilement dans la dynamique de chaque échange. Il y a déjà eu beaucoup de littérature sur la question et les limites imparties à cet article ne me permettaient pas de citer de larges portions de texte, seules à même de permettre l'observation de ces glissements. J'ai donc préféré me concentrer sur une question moins étudiée : pourquoi l'apostrophe apparaît-elle dans certains énoncés et non dans d'autres, alors que, non intégrée à la phrase, elle ne fait l'objet d'aucune contrainte syntaxique pesant sur son apparition ?

Dans cette première partie, je laisserai provisoirement de côté les apostrophes chargées d'une forte valeur appréciative et me limiterai à celles qui restent axiologiquement neutres et se bornent à désigner l'allocutaire par son nom, ou par un terme de parenté ou de politesse conventionnel. Je montrerai que ces apostrophes fonctionnent de façon très semblable à celles des discours témoins et jouent un double rôle de 
structuration du discours et de construction de la coénonciation, assez proche de celui qui a pu être dégagé pour les particules énonciatives ou les intonèmes par les travaux menés sur la structuration discursive à l'oral, notamment Roulet et al. (1985) et Morel et Danon-Boileau (1998).

\subsection{Structuration du discours et renforcement des actes illocutoires}

On observe tout d'abord que l'apostrophe apparaît préférentiellement en début et fin d'échange, pour signaler la reconnaissance réciproque des interlocuteurs, nouer le lien qui les unit et le réaffirmer au moment de la séparation :

1. Arrivé que je fus, la femme du Rey ne voulait pas me reconnaître : «Ça n'est pas possible que ce soit toi, Jacquou !» (Le Roy)

2. Enfin il se décida et se mit à parler d'un air vague, bredouillant un peu et regardant au loin dans la campagne. - Rose, dit-il, est-ce que tu n'as jamais songé à t'établir? (Maupassant)

3. Le lendemain matin, le curé, ayant emprunté la jument du chevalier, et ses houseaux, montait à cheval et partait pour Périgueux par les chemins de traverse, en passant par Saint-Geyrac. «Bon voyage, curé ! lui dit le chevalier » (Le Roy)

4. Et, m'étant habillé, je lui donnai le poignard de la demoiselle Galiote : « Gardez-moi cet outil, Jean, et au revoir !» (Le Roy)

Dans les exemples ci-dessus, l'apostrophe apparaît indifféremment dans l'énoncé en position initiale, médiane ou finale et permet au locuteur soit d'instituer son allocutaire comme tel en le nommant en début d'échange, soit de prendre congé de lui en fin d'échange. Cette fonction apparaît surtout dans les romans où il importe de bien démarquer le discours rapporté du récit et où les apostrophes en début de dialogue jouent un rôle équivalent de celui des didascalies théâtrales en précisant qui parle à qui, même en l'absence de discours attributif. On note ainsi qu'en (1) la phrase de récit qui précède ne constitue pas véritablement une annonce du discours rapporté mais plutôt un commentaire et qu'en (2) l'énoncé introductif ne précise pas à qui parle le personnage. L'apostrophe vient donc dans les deux cas combler une lacune en précisant le cadre énonciatif. En (3) et (4) l'énoncé rapporté clôt une des sections du chapitre sur une note dynamique tout en faisant l'économie d'un énoncé narratif qui décrirait les adieux du personnage. Dans le roman, le respect des règles conversationnelles qui passe, entre autres, par l'apostrophe à l'interlocuteur en début et fin d'échange, rend l'énoncé rapporté vraisemblable et celui-ci en retour contribue à la dynamique textuelle dans un étayage mutuel du récit et du discours où la mention des noms des personnages joue un rôle de balisage.

$\mathrm{Au}$ théâtre, du moins au $\mathrm{XX}^{\mathrm{e}}$ siècle, les échanges commencent rarement par des apostrophes: dans les trois pièces étudiées, les personnages ne s'interpellent jamais dans les premières répliques ${ }^{2}$. L'auteur laisse quelque temps planer un doute sur leur identité ou fait en sorte que des éléments verbaux ou non verbaux (costumes) permettent de les identifier sans qu'ils se nomment. En revanche, l'apostrophe accompagne systématiquement les changements d'allocutaires dans les scènes où figurent plusieurs personnages :

5. C'est bon, va-t'en, vieille ordure, et tâche de crever dans le repentir. C'est ta seule chance de salut. (La vieille s'enfuit.) Ou je me trompe fort, mes maîtres, ou voilà de la bonne piété, à l'ancienne, solidement assise sur la terreur. (Sartre)

Dans cette réplique des Mouches, la seconde apostrophe indique que le locuteur se tourne vers les personnages restés en scène et renoue avec eux le dialogue.

Le rôle de structuration discursive joué par l'apostrophe se manifeste aussi dans les discours que j'ai pris pour témoins. On la trouve en effet souvent dans des zones charnières, au début d'un nouveau développement, comme l'indiquent les exemples 6 à 8 où l'apostrophe accompagne des énoncés métadiscursifs gérant la progression textuelle : 
6. Après vous avoir exposé le premier effet de la grâce de Jésus-Christ en notre princesse, il me reste, messieurs, de vous faire considérer le dernier, qui couronnera tous les autres. (Bossuet)

7. Voilà, mes chers amis, l'essentiel de ce que j'avais à vous dire. (Blum, discours à la S.F.I.O.) [Cet énoncé marque le début de la dernière partie. Un peu plus loin, juste avant la conclusion, on trouve: Camarades, j'ai terminé.]

8. Messieurs, je ne reviens pas sur tout ce que j'ai dit. (...) Messieurs, j'ai achevé. (Blum, procès)

On remarque que dans les cas ci-dessus, où il s'agit de longs discours monologués, l'apostrophe se situe au début et à la fin d'une partie. On aurait pu s'attendre à ce que, dans les dialogues de théâtre, elle soit également assez présente en fin de réplique, pour indiquer à l'allocutaire que c'est son tour de parler. Or, il n'en est rien, et l'apparition de l'apostrophe au théâtre tient essentiellement à la nature de l'acte illocutoire effectué par l'énoncé : plus l'acte illocutoire est important, et plus il implique l'allocutaire, plus l'apostrophe se fait présente. C'est ainsi qu'il y a une forte corrélation entre l'apostrophe et les actes directifs, tout particulièrement avec les requêtes. Dans l'acte I scène 1 des Mouches, la longue tirade où Jupiter essaie de convaincre Oreste de quitter Argos est bâtie sur une alternance entre actes subordonnés assertifs et actes directeurs injonctifs. Or ce sont ceux-ci qui sont accompagnés d'apostrophes :

9. Les gens d'ici sont de grands pécheurs, mais voici qu'ils se sont engagés dans la voie du rachat. Laissez-les jeune homme, laissez-les, respectez leur douloureuse entreprise, éloignez-vous sur la pointe des pieds. (Sartre)

On trouve un fonctionnement similaire dans les discours témoins qui lient préférentiellement apostrophe et actes directifs :

10. Souvenez-vous donc, messieurs, de l'admiration que la princesse d'Angleterre donnait à toute la cour. Votre mémoire vous la peindra mieux avec tous ses traits et son incomparable douceur que ne pourront faire toutes mes paroles. (Bossuet)

L'apostrophe a également beaucoup d'affinité avec les énoncés assertifs contenant un acte argumentatif de premier plan (opposition à l'allocutaire, conclusion d'un raisonnement) :

11. Non, l'Afrique, ce n'est pas ce qu'on croit, bébé. (Koltès)

12. Alors, quand on vient ici, il n'est pas question de se laisser choir, non, bébé. (Koltès)

Quant aux énoncés interrogatifs, ils sont accompagnés d'une apostrophe surtout lorsqu'ils correspondent à un changement de thématique ou lorsque le locuteur oriente la conversation sur un terrain sensible :

13. CARMEN: Qu'est-ce que vous mettrez? IRMA: Le déshabillé crème. [didascalie décrivant l'habillage] Dis-moi, ma Carmen, Chantal ? CARMEN : Madame? IRma : Oui. Dis-moi, Chantal, qu'est-ce que tu sais d'elle? (Genet)

14. Avec cette aventure, pendant la guerre, Horn, avec cet... accident, il a dû gagner beaucoup d'argent, Horn; il n'en parle jamais, donc, ça doit être énorme. L'argent t'intéresse, hein, bébé ? (Koltès)

Dans les deux exemples ci-dessus, l'apostrophe resserre la relation entre les coénonciateurs au moment où la conversation change de sujet (ex. 13) et où le locuteur cherche à neutraliser la tentation éventuelle qu'aurait l'allocutaire de faire la sourde oreille (ex.14). Si l'on prend maintenant un exemple dans un roman, on voit que la longue plaidoirie de l'avocat, dans le procès relaté dans Jacquou le croquant, fait intervenir les apostrophes aux jurés (hormis le marquage du début et de la fin) en soutien des actes de langage les plus importants :

15. «Ah! il ne faut pas s'étonner, messieurs, que lorsque la justice et l'humanité sont ainsi outragées et violées impunément, la vindicte populaire s'élève et juge sommairement les coupables! (...) Messieurs les jurés, je 
Ces trois occurrences correspondent à l'argument-clé de l'avocat (les accusés n'ont fait que se substituer à la justice), et aux deux appels - indirect puis direct - aux jurés. De façon semblable, dans son procès, Blum, qui est très avare en apostrophes, en emploie une en soutien d'un acte illocutoire où il met toute sa légitimité dans la balance :

16. Messieurs, je vous assure que je suis bien là au cœur de la question ; j’y ai bien réfléchi. (Blum)

Dans tout le corpus il s'avère que, comme dans les discours témoins, l'apostrophe apparaît aux moments stratégiques de l'interaction, en appui des actes illocutoires directifs ou de ceux auxquels le locuteur veut donner le plus de poids. Dans la scène XIII de Combat de nègre et de chiens, Horn interpelle nommément Alboury lorsqu'il veut l'amener sur son terrain ou donner du poids à ses propres engagements en le prenant à témoin :

17. Reconnaissez que tous les pas, monsieur Alboury, c'est moi qui les ai faits : n'oubliez pas cela, quand nous récapitulerons. (Koltès)

18. Quand je vous dis, Alboury: faites-en ce que vous voulez je ne le couvre plus, ce n'est pas un mensonge, croyez-moi. (Koltès)

On trouve également l'apostrophe avec des actes promissifs (ex. 19) - comme si l'interpellation de l'allocutaire témoignait que le locuteur ne s'engage pas à la légère - ou avec des reproches impliquant l'allocutaire (ex. 20) :

19. Elle se rachètera, mon général. Je la connais. (Genet)

20. Ça n'est pas bien, Jacques, de me mépriser comme ça. (Maupassant)

L'apostrophe donne de la solennité à l'acte illocutoire : en réactivant le palier énonciatif sous-jacent à toute communication, elle atteste que le locuteur prend en compte son allocutaire et souhaite en retour qu'il le croie. Là encore les discours littéraires ne se séparent pas des autres discours. Lorsque Bossuet formule l'opinion paradoxale que la mort vaut mieux que la vie quand on détient le pouvoir et la gloire, il apostrophe son auditoire pour être sûr d'être suivi sur ce point délicat :

\footnotetext{
21. Mais ces idoles que le monde adore, à combien de tentations délicates ne sont-elles pas exposées ? (...) En cet état, messieurs, la vie n'est-elle pas un péril ? la mort n'est-elle pas une grâce ? (Bossuet)
}

Cet exemple montre que si l'apostrophe permet de soutenir les actes illocutoires importants ou ceux dont la réussite apparaît comme hasardeuse, elle est amenée à jouer un rôle de premier plan non seulement dans la structuration du discours mais aussi dans la gestion de l'interaction. Il convient alors de se demander si elle contribue plutôt à la construction du consensus ou si elle permet aussi d'opposer les deux personnes de l'énonciation à l'instar de la «déixis de rupture » que Danon-Boileau (1992: 421) opposait à la « déixis consensuelle».

\subsection{Apostrophe et marquage de l'altérité énonciative}

L'intervention du palier énonciatif dans la structure de l'énoncé remet au premier plan la relation sousjacente du locuteur et de l'allocutaire. Pour reprendre la terminologie de Jakobson (1963), à la centration sur le message, se substitue la centration sur le destinataire et l'accentuation de la fonction conative du langage, par exemple dans des actes de langage tels que les exhortations. On ne s'étonnera pas que, cherchant à galvaniser un groupe, celles-ci soient le plus souvent accompagnées de la mention explicite de l'allocutaire :

$$
\text { 22. «Courage, mes amis ! elle va céder !» m’écriai-je. (Le Roy) }
$$

Mais on aurait tort d'interpréter ces exhortations de façon fusionnelle. Le locuteur reste en quelque sorte sur l'autre rive du discours, distinct de ceux qu'il encourage. L'étude de mon corpus m'incite en effet à 
faire l'hypothèse que l'apostrophe surgit lorsque le locuteur veut marquer son propre positionnement face à un allocutaire auquel il propose, par l'apostrophe, un processus dynamique de différenciation débouchant selon les cas sur un éloignement ou un rapprochement ${ }^{3}$. Il peut sembler paradoxal d'activer la sphère du $t u$ lorsqu'on souhaite se positionner en tant que locuteur. C'est que ce positionnement ne doit pas être entendu au sens d'un recentrage égocentrique mais d'une recherche d'interaction (cf. Morel et Danon-Boileau 1998 : 94sq) : en marquant fortement la sphère du $t u$, le locuteur inscrit son dire dans un dialogue et par ricochet s'énonce autre, distinct de son allocutaire ${ }^{4}$. En signifiant à l'allocutaire qu'il fait bien partie de son horizon de discours, le locuteur lui impose par la même occasion la prise en compte réciproque de sa propre existence en tant que sujet doté d'un vouloir différent et éventuellement antagoniste $^{5}$. C'est pourquoi l'apostrophe se manifeste dans des situations d'interaction variées, allant de l'entente harmonieuse sur fond de dualité au conflit le plus marqué. Mais même dans ce dernier cas, elle est mouvement vers l'autre et marque un désir de convaincre, d'arracher le consensus, plutôt qu'un repliement dans la sphère du je.

Dans les dialogues d'amoureux, outre sa place privilégiée à l'ouverture et à la clôture de l'échange ${ }^{6}$, l'apostrophe apparaît lorsque l'échange prend un tour plus argumentatif, lorsque la tension vers l'autre et simultanément l'affirmation de soi se font plus fortes. Les exemples ci-dessous nous offrent différents contextes où le locuteur tour à tour signifie à l'allocutaire son étonnement admiratif, son désaccord, au moins verbal, ou l'altérité de son point de vue (en appui à mais, notamment) :

23. «Que tu es donc joliette, Lina ! - Tu dis ça pour rire, Jacquou! ( (Le Roy)

24. «Tu profites de ce que je suis trop bonne, Jacquou!» (Le Roy)

25. «Mais, ma chérie, je pensais que tu serais contente. Tu ne sors jamais, et c'est une occasion, cela, une belle ! (Maupassant)

C'est aussi l'altérité des points de vue que souligne dans Les Mouches l'emploi des apostrophes par le serviteur d'Oreste. Il n'y recourt que lorsqu'il est en désaccord avec son maître et cherche à obtenir de lui un revirement :

26. Ah ! mon maître, que vous me peinez ! Que faites-vous donc de mes leçons et de ce scepticisme souriant que je vous enseignai ? (Sartre)

27. Pas de souvenirs, maître ingrat, quand j'ai consacré dix ans de ma vie à vous en donner? (Sartre)

Les dialogues romanesques ou théâtraux, loin d'offrir une répartition régulière des apostrophes comme s'il s'agissait simplement de réactiver de temps en temps la fonction phatique chère à Jakobson, montrent bien que l'apostrophe correspond à des moments de tension interlocutive. Un bon exemple nous en est fourni par le récit du procès des paysans dans Jacquou le croquant: hormis les «monsieur le Président » rituels lorsqu'un des avocats demande la parole au juge, les apostrophes accompagnent des énoncés où le locuteur soit essaie d'influencer l'allocutaire soit marque son désaccord :

28. (...) elle dit nettement (...) qu'elle n'avait rien à me reprocher. « Pourtant, mademoiselle, répliqua le président, l'accusé Ferral avait des égratignures au visage, et vous-même aviez du sang sur la figure.(...) - Voyons, mademoiselle, peut-être éprouvez-vous quelque confusion, bien naturelle, à confesser cette tentative ; (...) dites-nous bien toute la vérité. - Je l'ai dite tout entière, monsieur. » (Le Roy)

29. Arrivez au déluge, maître Fongrave! dit le président qui, depuis le commencement de cette plaidoirie, s'agitait fiévreusement sur son fauteuil. - J'y suis, monsieur le Président ! (Le Roy)

L'énoncé du rang social, du titre, peut s'analyser un peu vite comme une marque de politesse, mais, outre que l'effet de politesse peut comme dans l'ex. 27 être annihilé par l'adjectif qui accompagne le titre, ou, comme dans l'ex. 29, fonctionner comme écho ironique, cette marque de politesse n'apparaît pas à chaque intervention mais uniquement dans des contextes à forte visée argumentative, ainsi qu'on peut le voir en (28) où le président pèse de tout son poids pour infléchir le témoignage de Mlle de Nansac, qui, de son côté, réaffirme sa position. 
Les travaux sur l'apostrophe, par exemple Lagorgette (2003: 63), ont déjà noté le rôle de soulignement de l'acte de langage que joue l'apostrophe, en interprétant généralement l'apostrophe comme un désir de renforcer le lien entre locuteur et allocutaire pour atténuer les face threatening acts (FTA) :

\section{[...] les T.A. [termes d'adresse] sont très fortement représentés dans les actes illocutoires : demande, ordre, mais aussi excuses, et généralement toute forme de négociation explicite. Les T.A. sont surtout utilisés par le locuteur dans des contextes de FTA afin de ménager la face de l'allocutaire.}

Mais il me semble qu'observer une coprésence entre FTA et apostrophe ne doit pas conduire nécessairement à penser que l'apostrophe n'est là que pour adoucir l'atteinte à la face positive ou négative de l'allocutaire. On peut au contraire faire l'hypothèse que, dans bien des cas, le marquage du palier énonciatif permet de souligner l'altérité des points de vue et aggrave les divergences, comme dans les ex. 26 à 29, au point, parfois, de déboucher sur un véritable duel verbal. J'en veux pour preuve que, dans le dialogue entre Irma et Carmen dans Le Balcon (cinquième tableau), c'est lorsque Carmen essaie de s'émanciper et de revendiquer son propre espace qu'elle ponctue ses énoncés d'un « madame Irma » :

30. Occupe-toi de mes additions. - Votre comptabilité ne remplacera jamais mon apparition. C'était devenu aussi vrai qu'à Lourdes. Maintenant tout en moi se tourne vers ma fille, madame Irma.(Genet)

Manière économique de dire «j'ai bien entendu votre requête mais je me positionne autrement», l'apostrophe intervient souvent en clôture d'un échange comme mesure d'une distance irréductible :

31. IRMA : Personne ne pourra donc y assister? Rien qu'une fois ? L'EVEQue, effrayé : Non, non, ces choses-là doivent rester et resteront secrètes. (...) Et que toutes les portes soient fermées. Oh, bien fermées, closes, boutonnées, lacées, agrafées, cousues... IRMA: Je vous le demandais... L'EVEQUE : Cousues, madame Irma. (Genet)

On voit dans l'exemple 31 que, malgré l'insistance d'Irma qui réitère sa demande, l'évêque met un terme à la discussion au moyen de l'apostrophe. Celle-ci, en prenant l'allocutaire à témoin du non-désir du locuteur d'accéder à sa requête, fait d'une pierre deux coups : elle réinscrit l'autre dans le discours au moment où s'affirme l'intransigeance du locuteur et sa supériorité pragmatique.

Si le signifié du SN mis en apostrophe est souvent vecteur d'une certaine politesse rituelle, et parfois d'un sentiment d'affection, l'acte même de l'apostrophe doit à mon sens être dissocié de ce signifié conjoncturel pour être envisagé de façon plus abstraite comme un souci du locuteur de se positionner comme distinct de son allocutaire. L'apostrophe me semble avoir partie liée avec l'affirmation dans le discours de l'altérité du je par rapport au $t u$, altérité qui, selon les cas, peut se décliner sur le mode de l'échange amical ou amoureux, du duel oratoire, de l'insulte ${ }^{7}$. A la suite de Détrie (2006: 165), j'incline ainsi à voir dans l'apostrophe un des signes du passage à la sphère de la subjectivité en soi-même dans le processus d'actualisation discursive tel que l'envisage la praxématique :

\footnotetext{
Le stade 3 [de l'actualisation discursive] correspond à l'ego (...) En un sens, il est le comble de la subjectivité. En un autre sens, il résulte de l'objectivation de soi. Le sujet se conçoit comme autre de l'autre, et doit admettre que chaque être humain, comme lui, a droit aux privilèges de la subjectivité. Cette relativité de je et l'objectivation de soi qu'elle implique conduisent à une organisation stricte de la sphère interpersonnelle et déictique, selon le mode de l'opposition et de la rupture entre les instances. La personne s'oppose à la non-personne, et à l'intérieur de la personne s'opposent $j e$ et $t u$. (Détrie, Siblot, Verine $2001: 332$ )
}

On peut d'ailleurs considérer que les rôles de structuration du dialogue et de soutien des actes illocutoires directeurs que j'ai assignés précédemment à l'apostrophe constituent d'autres manifestations de ce même marquage de l'altérité dans des contextes spécifiques où il importe de (re)préciser les cadres de l'échange, soit pour faciliter le repérage par l'auditoire des moments sensibles que sont l'ouverture, le changement de parties, ou la clôture, soit pour s'assurer de son implication lors d'un acte illocutoire décisif. 
En tous les cas, la scène énonciative (Maingueneau 2004) romanesque et théâtrale, pour fictive qu'elle soit, ne se distingue pas ici de l'usage habituel de l'apostrophe dans d'autres discours effectivement prononcés devant un auditoire.

\section{L'apostrophe en poésie}

Dans cette exploration des fonctions de l'apostrophe, j'ai laissé de côté pour l'instant les textes poétiques de mon corpus car, dans ces textes, l'apostrophe ne s'inscrit pas dans un cadre dialogal préexistant, mais contribue au contraire à faire du poème le lieu d'un échange dialogué entre le locuteur et les entités qu'elle désigne ${ }^{8}$. L'investigation du corpus se fera donc selon deux axes :

- je montrerai tout d'abord en quoi la scène énonciative propre à la poésie implique un usage spécifique de l'apostrophe ;

- j'indiquerai ensuite les parentés entre l'usage poétique de l'apostrophe et certains usages observables dans d'autres discours, ce qui me permettra de ne pas isoler le discours poétique dans une irréductible singularité.

\subsection{Entre allocutif et élocutif}

Un premier exemple assez simple, extrait du Roman inachevé d'Aragon, permettra d'illustrer la possibilité dans le discours poétique d'instituer très facilement une non-personne en personne à laquelle on s'adresse par l'introduction d'une apostrophe :

\section{Regardez les jeunes gens avec ce qu'ils traînent / La superstition qui s'attache à leurs pas (...) // Regardez ces jeunes gens Qu'est-ce qui les pousse / Comme ça vers les bancs de sable les bas-fonds (...) // Bon Dieu regardez-vous petits dans les miroirs/ Vous avez le cheveu désordre et l'œil perdu (Aragon)}

Au fil des trois strophes, pour chacune desquelles je reproduis les deux premiers vers, les « jeunes gens » regardés par le locuteur et son lecteur sont attirés dans la sphère de l'interlocution par le biais du démonstratif « ces » puis interpellés par le locuteur et deviennent donc les interlocuteurs du poète, avant de redevenir deux strophes plus bas des délocutés. Non soumise aux contraintes du réalisme, la poésie offre une grande labilité de la scène énonciative. Certains morphèmes qui sont devenus des indices du poétique contribuent à cette labilité. Tel est tout particulièrement le cas de $\hat{o}$ qui précède aussi bien des apostrophes que des énoncés exclamatifs (cf. Détrie 2006 : 169-171) et assure fréquemment le passage entre un énoncé dominé par ce que Charaudeau (1992 : 574) appelle un acte « allocutif » où le locuteur se positionne par rapport à un interlocuteur qu'il "implique » dans son acte d'énonciation, et un énoncé dominé par un acte « élocutif» où le locuteur « situe son propos par rapport à lui-même » (ibid.). Dans un genre de discours qui ne peut s'appuyer, comme c'est le cas dans la représentation théâtrale ou dans la fiction romanesque, sur les bénéfices de la mimésis, le rôle de $\hat{o}$ me semble avant tout, de par sa valeur déixisante que j'ai décrite dans un article antérieur (Monte 2005), de contribuer à créer de toutes pièces une situation d'énonciation qui présentifie les référents. Mais, par ailleurs, sa capacité à introduire des SN en apostrophe aussi bien que des SN exclamatifs dans des énoncés averbaux a pour effet secondaire d'augmenter le brouillage entre acte élocutif et allocutif caractéristique de maints textes poétiques et facilité par les incertitudes de la syntaxe et de la ponctuation :

33. L'amour durcit tes baies sauvages, ô terre plus crépelée que le chagrin des Maures! ô mémoire, au cœur d'homme, du royaume perdu! (Saint-John Perse)

34. Destins destins impénétrables / Rois secoués par la folie / Et ces grelottantes étoiles / De fausses femmes dans vos lits / Aux déserts que l'histoire accable (Apollinaire) 
En (33), si le premier syntagme nominal «ô terre plus crépelée que le chagrin des Maures » s'interprète comme apostrophe en raison de la corréférence avec le déterminant «tes », il est loisible au lecteur de considérer le second syntagme nominal (ici en italiques) comme un énoncé averbal exclamatif commentant l'énoncé antérieur ou comme une deuxième apostrophe, sans énoncé-hôte. En (34), le début de la strophe privilégie l'acte élocutif d'appréciation (Charaudeau 1992:605) mais la présence ensuite du déterminant « vos » tend à faire réinterpréter rétroactivement « rois secoués par la folie » comme une apostrophe. Dans les deux cas, on voit que s'établit une tension entre une structure apparente (au niveau rythmique et syntaxique) qui privilégie la reprise du même et une structure virtuelle qui introduit de la différenciation. Cette tension, qui naît ici de la contestation possible de l'équivalence fonctionnelle entre deux syntagmes successifs, peut d'autres fois résider au cœur d'un seul syntagme nominal qui superpose en lui-même les deux possibilités interprétatives :

\section{Oiseau tranquille au vol inverse oiseau / Qui nidifie en l'air / À la limite où notre sol brille déjà / Baisse ta deuxième paupière la terre t'éblouit / Quand tu lèves la tête (Apollinaire) \\ 36. Temps passés Trépassés Les dieux qui me formâtes / Je ne vis que passant ainsi que vous passâtes (Apollinaire)}

La présence dans ces deux énoncés de marques allocutives très fortes - l'impératif «baisse » et les trois marques de personne 2 en (35), la désinence de personne 5 placée à la rime et le pronom « vous » en (36) - incite à une lecture comme apostrophe préphrastique des syntagmes en italiques mais à l'inverse l'absence de désinence de personne 2 à «nidifie » et la présence de l'article «les » devant «dieux» incline à y voir pour (35) un énoncé averbal existentiel (Lefeuvre 1999) et en (36) le thème postposé du rhème "trépassés» dans un énoncé averbal à deux termes. On a donc l'impression d'une syntaxe mouvante, qui joue avec les normes orthographiques ou syntaxiques pour permettre une modification perpétuelle de la scène énonciative : tel élément posé tout d'abord dans son existence indépendamment de la relation interlocutive se trouve ensuite brusquement inclus dans cette relation au titre d'allocutaire. A l'inverse la relation interlocutive peut se distendre comme en (33) au profit d'un recentrage sur l'émotion du locuteur, et ce d'autant plus aisément que l'allocutaire est un non-humain promu coénonciateur par la seule vertu de l'écriture.

Très fréquente dans les trois recueils étudiés, l'apostrophe se trouve ainsi à la fois exaltée et menacée, puisque, tout en faisant du poème le lieu d'une énonciation interactive très marquée, elle est en même temps toujours susceptible de se résorber dans un énoncé affectif appréciatif où l'allocutaire devient pur délocuté. Cette particularité de l'apostrophe en discours poétique trahit en quelque sorte la nature fictive de l'allocution qui s'y montre et qui n'a d'autre existence que celle que lui donne le poème. Là où le roman ou le théâtre s'adossent dans un rapport mimétique à la communication ordinaire et en épousent les exigences, la poésie lyrique, en s'affranchissant de ces contraintes, met au centre de son discours un locuteur en quête de dialogue mais limité à des allocutaires éphèmères et généralement muets.

\subsection{Apostrophes caractérisantes}

L'autre particularité de l'apostrophe dans les textes poétiques est qu'au lieu de désigner des entités déjà stabilisées par le contexte, elle les introduit dans le discours. De ce fait elle remplit aussi un rôle très important de caractérisation. L'oiseau de (35) et les dieux de (36) apparaissent le premier au tout début du poème, le second en début de strophe, et c'est dans un même énoncé qu'ils sont à la fois intronisés comme allocutaires et introduits comme supports de prédicats descriptifs ou actionnels. Ceci semble de prime abord très spécifique du texte poétique, si l'on compare avec les syntagmes que nous avons rencontrés précédemment et qui se bornaient à désigner les référents par leur prénom ou nom patronymique ou par un titre renvoyant de façon stable à leur rôle social ou à leur position par rapport au locuteur. Voici quelques exemples de ces caractérisations où les expansions adjectivales et les appositions jouent un rôle important :

37. Bergère ô tour Eiffel le troupeau des ponts bêle ce matin (Apollinaire)

38. Malheur dieu pâle aux yeux d'ivoire / Tes prêtres fous t'ont-ils paré / Tes victimes en robe noire / Ont-elles vainement pleuré / Malheur dieu qu'il ne 
faut pas croire // Et toi qui me suis en rampant / Dieu de mes dieux morts en automne / Tu mesures combien d'empans / J'ai droit que la terre me donne / $\hat{O}$ mon ombre ô mon vieux serpent (Apollinaire)

39. Ô mort parée du gantelet d'ivoire, tu croises en vain nos sentes bosselées d'os, car notre route tend plus loin. (Saint-John Perse)

40. Nous en avions assez du doigt de craie sous l'équation sans maître... Et vous, nos grands Aînés, dans vos robes rigides, qui descendez les rampes immortelles avec vos grands livres de pierre, nous avons vu remuer vos lèvres dans la clarté du soir : vous n'avez dit le mot qui lève ni nous suive. (Saint-John Perse)

41. Ô part ! ô réservée ! ô inspiratrice ! ô partie réservée de moi-même ! ô partie antérieure de moi-même ! / Ô idée de moi-même qui étais avant moi ! / $O$ partie de moi-même qui es étrangère à tout lieu et ma ressemblance éternelle qui / Touches à certaines nuits / Mon cœur (...) / Vois-moi, ridicule et blessé, étouffé au milieu de ces hommes irrespirables, $\hat{o}$ bienheureuse, et dis une parole céleste! (Claudel)

42. Fils de nos os chrétiens, aie compassion de notre pourriture, dont Dieu a dit qu'elle est ta mère et tes frères ! (...) / Ô vivant qui peux encore mériter ! $\hat{o}$ possesseur d'inépuisables richesses! (Claudel)

Dans tous ces exemples, l'apostrophe en position préphrastique introduit un nouveau référent dans le discours : elle joue donc le rôle, comme les dislocations gauche, d'introducteur de topique. La contrainte d'accessibilité pragmatique des topiques (Lambrecht 1998 : 39), qui est a priori satisfaite dans le cas des apostrophes par le fait qu'il s'agit de l'allocutaire présent dans la situation d'énonciation, se trouve ici préservée par le caractère présupposé des désignations : la caractérisation est présentée comme un acquis antérieur du discours, selon le principe général d'évocation qui régit l'énonciation poétique (Dominicy 1992). Les adjectifs substantivés ou les substantifs dotés d'expansions descriptives sont censés non pas asserter une identité mais évoquer une entité déjà connue du lecteur. On pourrait penser qu'il ne s'agit là que d'un artifice discursif résultant des spécificités de la communication littéraire, comme nous le rappellent Détrie et Verine $(2003: 218)$ :

La communication littéraire obéit à l'enchâssement de deux interactions : d'une part, l'interaction enchâssante, représentante, du scripteur avec le lecteur dans le contexte social du marché littéraire ; d'autre part, l'interaction représentée, enchâssée, entre les voix mises en spectacle par le texte.

Et certes, on envisage mal dans la communication ordinaire que le locuteur décrive longuement son allocutaire avant de formuler l'acte de langage qui a suscité l'interpellation. La sobriété des apostrophes dans le reste de notre corpus, qui se veut mimétique de cette communication ordinaire, contraste d'ailleurs vivement avec ces débauches de caractérisation. Pourtant, il ne me semble pas impossible de rapprocher ces énoncés poétiques d'un usage bien attesté de l'apostrophe dans tous les genres discursifs et qu'illustrent les exemples ci-dessous empruntés aux romans de mon corpus :

43. Moi, oyant cette bête-là parler ainsi du curé, je fus au moment de lui crier sur le coup de la colère qui me monta : «Tu en as menti, gredin!» (Le Roy)

44. « Soyez le très bien venu, mon vieil ami !» (Le Roy)

45. «Foutu gueux! ma drole est perdue par la faute de ton coquin de fils : tu vas payer pour lui !» Et à cette voix s'en joignaient d'autres [...] levant les bras, je criai : "Arrêtez !... Jusqu'ici, braves gens, je vous ai bien conseillés, n'est-ce pas ? Eh bien, écoutez-moi encore !» (Le Roy)

46. Le malheureux criait comme un porc qu'on va saigner, ne s'interrompant que pour demander grâce d'une voix piteuse. «Allons, tais-toi, braillard! ne vois-tu pas tous les autres sur pied ?...» (Le Roy)

En même temps qu'elle interpelle, l'apostrophe a ici pour fonction de qualifier l'allocutaire de façon positive ou négative. Le lien avec l'énoncé-hôte est ici très proche de ce qui se passe dans le cas de l'apposition": une relation logique s'établit, soit que l'acte de parole effectué au moyen de l'énoncé découle de la qualité attribuée à l'allocutaire (ex. 44, 46), soit, à l'inverse, que l'énoncé justifie l'insulte 
ou l'éloge (ex. 43 et première occurrence de 45), soit, enfin, qu'il s'efforce de recueillir les bénéfices de la désignation opérée, comme dans la deuxième occurrence de 45 où Jacquou qualifie de «braves gens » les paysans dont il veut calmer la colère. Par ailleurs, dans les romans, l'apostrophe entretient aussi un lien sémantique avec les actions relatées dans le récit enchâssant le discours rapporté : elle apparaît ainsi doublement déterminée.

Dans les énoncés poétiques, on observe la même solidarité entre l'apostrophe et l'énoncé-hôte. Le cas est particulièrement flagrant dans le premier exemple d'Apollinaire où c'est la désignation de la tour Eiffel comme bergère qui justifie l'énoncé « le troupeau des ponts bêle ce matin » selon le processus de la métaphore filée. L'énoncé-hôte déroule ainsi le programme sémantique contenu dans le signifié du SN. On observe aussi que les apostrophes sont souvent redoublées, de sorte qu'un même référent se trouve successivement nommé puis caractérisé (ainsi le « malheur» dans l'ex. 38) ou bien présenté à la fois sous son nom et sous une désignation métaphorique (ainsi de l'ombre, toujours en 38). Mouvante et hypertrophiée, l'apostrophe prend en charge l'évolution dans la façon de considérer le référent et devient un des lieux cruciaux où se formule le point de vue du locuteur. Elle peut, comme dans les cas usuels d'insulte ou d'éloge, appuyer l'acte de langage formulé dans l'énoncé-hôte : ainsi, en (41) et (42), les caractérisations de l'âme et des vivants effectuées au moyen des apostrophes justifient-elles les supplications qui leur sont adressées et présagent-elles leur exaucement. Mais le rôle principal de l'apostrophe est de donner corps à des entités en leur conférant l'évidence des descriptions présupposées et en permettant au lecteur de se les imaginer.

Ainsi l'apostrophe poétique caractérisante obéit-elle à deux nécessités complémentaires : d'une part, comme les apostrophes appréciatives des autres discours littéraires ou des discours quotidiens, elle permet l'expression du point de vue du locuteur et légitime ses actes illocutoires; d'autre part, elle prend en compte les nécessités d'une scène énonciative spécifique où le locuteur interpelle des entités qu'il est censé connaître mais que le lecteur, lui, ignore, puisqu'elles n'ont pas encore été introduites dans le discours. L'hypertrophie des apostrophes correspond alors à une interlocution fictive où le rôle d'ordinaire dévolu aux énoncés descriptifs se trouve assumé par les interpellations. L'apostrophe cumule en effet deux avantages contradictoires: elle apparaît comme porteuse d'une mémoire de l'univers discursif qu'elle présente comme déjà là avant sa profération et simultanément elle montre les éléments de cet univers discursif dans leur surgissement sur la scène du poème. L'indiscutable évidence des référents des $\mathrm{SN}$ en apostrophe tient aux effets conjugués de la présupposition attachée à la référence définie et de la deixis ad oculos, d'où l'étonnante présence de ces entités qui ne doivent pourtant leur existence qu'au texte même. Celui-ci affiche ainsi dans l'usage des apostrophes le pouvoir non seulement de nomination mais aussi de convocation de l'énoncé poétique dès lors qu'il imite les conditions de l'échange oral direct sans abdiquer la possibilité de décrire en même temps qu'il interpelle.

On aurait tort cependant d'isoler ce fonctionnement de l'apostrophe caractérisante en poésie de l'emploi plus général de l'apostrophe appréciative dont l'insulte constitue un cas particulier, déjà bien étudié (cf. note 5). Simplement, alors que les bénéfices de l'apostrophe appréciative sont en général de type $\operatorname{argumentatif}^{10}$, en poésie ils sont surtout référentiels et textuels : combinant description et construction de la scène énonciative, l'apostrophe concourt à la densité de l'énoncé poétique autant qu'à sa puissance évocative et place le locuteur au centre d'un réseau interlocutif multiple où son rôle d'interpellant n'est pas sans lien avec son ancienne position de vates.

Nous pouvons donc conclure que, dans les situations de communication spécifiques que sont les trois formes du discours littéraire envisagées ici, l'apostrophe remplit un rôle énonciatif qui est aussi le sien dans des discours non fictifs. A ce titre, l'étude des discours littéraires nous éclaire sur des phénomènes fondamentaux tels que la construction de l'altérité lors du processus discursif. L'apostrophe, jamais obligatoire et donc toujours signifiante, fournit un bon lieu d'observation pour étudier le déroulement d'échanges qui, bien que surdéterminés par leur scène énonciative, satisfont néanmoins aux règles pragmatiques de l'interlocution. Mais, simultanément, l'usage de l'apostrophe montre aussi comment le discours littéraire peut utiliser certaines formes pour renforcer la cohésion textuelle entre éléments hétérogènes tels que le récit et le discours rapporté et, dans certains cas, hypertrophier une des fonctions des formes utilisées pour construire une scène énonciative spécifique. 


\section{Corpus}

(La date qui figure entre parenthèses est celle de l'édition dont je me suis servie, suivie entre crochets de celle de la première publication)

Apollinaire G., Alcools (1972, [1913]), p. 1 à 83, coll. Poésie, Gallimard, Paris.

Aragon L., Le Roman inachevé (1972, [1956]), première partie, coll. Poésie, Gallimard, Paris.

Blum L., Discours (1997), chap. VII, VIII et XII, Imprimerie Nationale, Paris.

Bossuet J.-B., Oraisons funèbres (2004, [1689]), Petits classiques Larousse, Paris.

Claudel P., Cinq grandes odes, (1970, [1957]), « La Muse qui est la grâce » (quatrième ode), coll. Poésie, Gallimard, Paris.

Genet J., Le Balcon (1979, [1956]), tableaux 1 à 3, coll. Folio, Gallimard, Paris.

Koltès B.-M., Combat de nègre et de chiens (1996, [1989]), sc. I à XIV, éd. de Minuit, Paris.

Le Roy E., Jacquou le Croquant (1990, [1900] ), chap. 5 et 7, Presses Pocket, Paris.

Maupassant G., «Boule de Suif» (1880), «Histoire d'une fille de ferme » (1881), «La Parure » (1884), «L'Aveu » (1884) dans Boule de Suif, La Maison Tellier et autres contes (1973), coll. Folio, Gallimard, Paris.

Saint-John Perse, Chronique (1968, [1959]), dans Vents suivi de Chronique, coll. Poésie, Gallimard, Paris.

Sartre J.-P., Les Mouches (1972, [1947]), acte I, coll. Folio, Gallimard, Paris.

\section{Références bibliographiques}

Charaudeau, P. (1992). Grammaire du sens et de l'expression. Paris : Hachette.

Danon-Boileau, L. (1992). «Ce que "ça” veut dire : les enseignements de l'observation clinique » dans La deixis, Morel, M.A. et Danon-Boileau, L. (éds.), Paris : PUF, p. 415-425.

Détrie, C. (2006). De la non-personne à la personne : l'apostrophe nominale. Paris : CNRS éditions.

Détrie, C. et Vérine, B. (2003). "Modes de textualisation et production du sens : l'exemple de "Complainte d'un autre Dimanche" de Jules Laforgue », dans L'analyse du discours dans les études littéraires, Amossy et Maingueneau (dir.), Toulouse : Presses Universitaires du Mirail, p. 213-226.

Détrie, C., Siblot, P. et Verine, B. (2001). Termes et concepts pour l'analyse du discours. Une approche praxématique. Paris : Champion.

Dominicy, M. (1992). «Pour une théorie de l'énonciation poétique », dans Enonciation et parti-pris, W. de Mulder, F. Schuerewegen et L. Tasmowski (éds), Amsterdam : Rodopi, p. 129-142.

Jakobson, R. (1963, trad. fr. [1960]). Essais de linguistique générale. Paris : Minuit.

Lagorgette, D. (2003). «Termes d'adresse, insulte et notion de détachement en diachronie : quels critères d'analyse pour la fonction d'adresse? », Cahiers de praxématique 40, p. 43-70, Montpellier.

Lagorgette, D. et Larrivée, P. (éds.) (2004). «Les insultes: approches sémantiques et pragmatiques », Langue française 144. Paris : Larousse..

Lambrecht, K. (1998). «Sur la relation formelle et fonctionnelle entre topiques et vocatifs », Langues, 1, p. 34-45.

Lefeuvre, F. (1999). La phrase averbale en français. Paris : L'Harmattan.

Maingueneau, D. (2004). Le discours littéraire. Paris : Armand Colin.

Monte, M. (2005). « Ô + SN dans les textes poétiques : entre apostrophe et exclamation », Modèles linguistiques, tome XXVI-2, année 2005, vol. 52, p. 45-68.

- (2007). «Tension énonciative et cohésion textuelle dans Vents, Chronique et Chant pour un équinoxe : le rôle des vocatifs », in Questions de style, $\mathrm{n}^{\circ} 4$, revue électronique de l’Université de Caen, Laure Himy (éd.), p.61-79, http://www.unicaen.fr/services/puc/revues/thl/questionsdestyle/ 
- (à paraître). «L'apostrophe dans les textes littéraires : prédicativité et valeurs illocutoires », dans Les linguistiques du détachement, Actes du colloque de Nancy de juin 2006, Apothéloz D., Combettes B. et Neveu F. éds, Berne : Peter Lang.

Morel, M.A. (2003). «Fusion / Dissociation des points de vue dans le dialogue oral : intonation et syntaxe discursive ", Cahiers de praxématique 41, p. 157-190, Montpellier.

Morel, M.A. et Danon-Boileau, L.(1998). Grammaire de l’intonation. Paris-Gap : Ophrys.

Neveu, F. (1999). «Vocatifs et formats syntaxiques dans Stèles », dans Phrases : syntaxe, rythme, cohésion du texte, Neveu F. (éd.) Paris : SEDES, p. 277-295.

Perret, D. (1970). «Les appellatifs : analyse lexicale et actes de parole », Langages 32, p. 112-118, Paris : Larousse.

Roulet E. et al. (1985). L'articulation du discours en français contemporain, Berne : Peter Lang.

\footnotetext{
${ }^{1}$ Laboratoire BCL, Université Nice Sophia-Antipolis, CNRS ; MSH de Nice, 98 bd E. Herriot, 06200 NICE

2 Une comparaison avec des dialogues oraux spontanés montrerait peut-être que le dialogue romanesque est finalement plus proche de l'usage spontané que le théâtre.
}

3 On pourrait parler en termes culioliens d'un repérage par disjonction.

4 L'oralisation des énoncés étudiés permettrait sans doute de vérifier par l'intonation ces hypothèses sur la construction de la coénonciation au niveau de l'apostrophe.

5 Morel (2003 : 170) observe aussi dans un dialogue oral que l'apostrophe apparaît dans le préambule lorsque «le parleur se place de son point de vue à lui », le préambule décondensé permettant à la fois de rechercher un consensus et d'affirmer un point de vue personnel sur le référent.

6 Il s'agit alors de se reconnaître mutuellement comme objet d'amour et de le réaffirmer au moment de la séparation.

7 Je ne m'attarderai pas sur l'insulte car c'est un domaine qui a déjà été bien balisé par les travaux sur l'apostrophe (cf. entre autres Lagorgette et Larrivée éds 2004 et Détrie 2006 : 142-152).

8 J'ai montré ailleurs (Monte 2007) que, dans certains poèmes contemporains, le locuteur ne prend consistance qu'à partir du moment où il interpelle des instances à vocation légitimante, dans un mouvement qui prolonge la traditionnelle invocation à la Muse des poètes de l'Antiquité.

9 Sur les liens et les différences entre apostrophe et apposition, on pourra consulter Neveu (1999) et Monte (2005 et à paraitre).

10 Détrie écrit à ce sujet : «L'apostrophe non seulement efface l'articulation argumentative, mais aussi pose la caractérisation effectuée comme syntaxiquement irréfutable, en cumulant référenciation et qualification » (2006: 137). 